\title{
Variational Convergence of Finite Networks
}

\author{
Atsushi KASUE* \\ Department of Mathematics, Kanazawa University, Kanazawa 920-1192, Japan
}

\begin{abstract}
We report some recent results and raise some open questions concerning Gromov-Hausdorff, variational and spectral convergence of finite networks endowed with the resistance metrics and measures, and also Royden's compactification of infinite networks.
\end{abstract}

Mathematics Subject Classifications (2000): 53C21, 58D17, 58J50.

KEYWORDS: networks, resistance form, resistance metric, convergence of Gromov-Hausdorff sense, $\Gamma$-convergence, Royden's compactification

\section{Introduction}

A Dirichlet problem on harmonic functions in a relatively compact domain of a Riemannian manifold is solved by the direct method of calculus of variations as follows. We first take a sequence of functions with prescribed boundary values which is minimizing the Dirichlet energy. Then it follows from the Poincare inequality that the sequence is bounded in the Sobolev space, and hence by Rellich's theorem, we have a subsequence which converges to a function weakly in the Sobolev space and strongly in $L^{2}$ space. The lower semi-continuity of the Dirichlet energy implies that the limit function minimizes the energy and satisfies the Laplace equation in a weak sense. Secondly we derive $a$ priori estimates on the Hölder continuity of the solution with respect to the Riemannian distance, using Moser's iteration method in which the volume estimates of metric balls, the Sobolev inequality, and Poincaré inequality play crucial roles.

Riemannian manifolds are metric spaces with the Riemannian distances and also Dirichlet spaces with the Dirichlet energy functionals on the Sobolev spaces. From the former point of view, the convergence of Riemannian manifolds in the Gromov-Hausdorff sense has been intensively studied. We have some survey articles, for instances, [8, 28, 29] on the collapsing phenomena, [22] on the convergence of Einstein manifolds, [3,24] on the Riemannian manifolds with Ricci curvature bounded below. Also we can refer to the monographs $[1,10,11]$ for discussions in details. On the other hand, from a view point of the direct method of calculus of variations mentioned above, the convergence of the Dirichlet energy functionals on Riemannian manifolds and more generally certain Dirichlet spaces are studied in $[15,19,21]$ (see [16]).

In this paper, we first report some recent results concerning variational convergence of finite networks. Finite networks are regarded as subspaces of metric graphs, by which we mean Riemannian polyhedra of dimension one ( $c f$. [6]). Two metric structures can be introduced on a metric graph, from the geodesic distance and from the effective resistance which is associated with a canonical energy form of the graph, called the resistance form. Accordingly, we are able to discuss convergence of metric graphs with respect to the Gromov-Hausdorff distance. Any compact geodesic space, for instance, turns out to be the limit of compact metric graphs endowed with the Riemannian distances. On the other hand, a certain class of so called fractal sets including the Sierpinski gasket has been studied as limits of finite networks with the resistance metrics by J. Kigami ( $c f$. [20] and the references therein).

An infinite network may be viewed as a limit of finite ones and also considered as a combinatorial approximation of noncompact Riemannian manifolds. In the final section, we make some observations on Dirichlet finite harmonic functions and points at infinity of an infinite network, raising some open questions.

The details of the results mentioned in this report will be taken up in [17] and [18].

\section{Metric Graphs, Subspaces and Energy Forms}

We are given a finite graph $(V, E)$ with the sets of verticies $V$ and edges $E$ and a positive function $r$ on $E$. Loops and multiple edges are admitted. Then by regarding edges $e$ as curves of length $r(e)$ and gluing them at the verticies, we obtain a compact metric graph, that is, a compact Riemannian polyhedron $G$ of dimension one. We suppose that $G$ is connected unless otherwise are stated. We denote by $d_{G}^{R}$ and $s_{G}$ the Riemannian distance and the canonical Riemannian measure of $G$, respectively.

An energy form on the space of continuous functions on $G, C(G)$, equipped with the uniform norm \|\|$_{C^{0}}$ is defined as follows. Let $D\left[\mathcal{E}_{G}\right]$ be the space of functions $u \in C(G)$ such that on each edge (or one-simplex) $e$ of $G$ which is parametrized by the arc length in the interval $[0, r(e)], u$ is absolutely continuous and the derivative $u^{\prime}$ belongs to

* Partly supported by the Grant-in-Aid for Scientific Research (B) No. 15340053 of the Japan Society for the Promotion of Science 
$L^{2}([0, r(e)])$. Then a canonical energy form $\mathcal{E}_{G}$ on $D\left[\mathcal{E}_{G}\right]$ is given by

$$
\mathcal{E}_{G}(u, v)=\int_{G} u^{\prime} v^{\prime} d s_{G}=\sum_{e \in E} \int_{0}^{r(e)} u^{\prime}(s) v^{\prime}(s) d s, \quad u, v \in D\left[\mathcal{E}_{G}\right] .
$$

Let

$$
R_{G}(x, y)=\sup \left\{\frac{|u(x)-u(y)|^{2}}{\mathcal{E}_{G}(u, u)} \mid u \in D\left[\mathcal{E}_{G}\right], \mathcal{E}_{G}(u, u) \neq 0\right\}, \quad x, y \in G .
$$

The number $R_{G}(x, y)$ is called the effective resistance between the points $x$ and $y$ of $G$.

The form $\varepsilon_{G}$ has the following properties.

(i) $\mathscr{E}_{G}$ is symmetric and positive semi-definite, and $\mathscr{E}_{G}(u, u)=0$ if and only if $u$ is constant.

(ii) The form $\varepsilon_{G}(u, v)+u(o) v(o)$ on $D\left[\varepsilon_{G}\right]$, where $o$ is a fixed point of $G$, is closed in the sense that it provides a complete inner product on $D\left[\mathcal{E}_{G}\right]$.

(iii) For a sequence of functions $u_{n}$ in $D\left[\varepsilon_{G}\right]$ whose energies $\varepsilon_{G}\left(u_{n}, u_{n}\right)$ are uniformly bounded, if $u_{n}$ uniformly converges to a function $u$ in $C(G)$ as $n \rightarrow \infty$, then we have

$$
\varepsilon_{G}(u, v)=\lim _{n \rightarrow \infty} \varepsilon_{G}\left(u_{n}, v\right)
$$

for all $v \in D\left[\mathcal{E}_{G}\right]$. As a result, the energy form $\mathcal{E}_{G}$ is lower semi-continuous on $C(G)$, that is, for a sequence of functions $u_{n} \in C(G)$ which uniformly converges to a function $u \in C(G)$, we have

$$
\mathcal{E}_{G}(u, u) \leq \liminf _{n \rightarrow \infty} \mathcal{E}_{G}\left(u_{n}, u_{n}\right) \quad(\leq+\infty) .
$$

(iv) A function $u \in D\left[\mathcal{E}_{G}\right]$ satisfies

$$
|u(x)-u(y)|^{2} \leq \mathcal{E}_{G}(u, u) d_{G}^{R}(x, y), \quad x, y \in G,
$$

and hence by the definition of $R_{G}$, this estimate is restated as follows:

$$
\begin{gathered}
|u(x)-u(y)|^{2} \leq \mathcal{E}_{G}(u, u) R_{G}(x, y), \quad u \in D\left[\mathcal{E}_{G}\right], \quad x, y \in G ; \\
R_{G}(x, y) \leq d_{G}^{R}(x, y), \quad x, y \in G .
\end{gathered}
$$

In particular, we have

$$
|u(x)| \leq \mathcal{E}_{G}(u, u)^{1 / 2} R_{G}(x, y)^{1 / 2}+|u(y)|, \quad x, y \in G .
$$

(v) $\mathscr{E}_{G}$ satisfies the Markov property, that is, for $u \in D\left[\mathcal{E}_{G}\right], \bar{u}:=\min \{\max \{0, u\}, 1\} \in D\left[\mathcal{E}_{G}\right]$ and

$$
\varepsilon_{G}(\bar{u}, \bar{u}) \leq \mathcal{E}_{G}(u, u) .
$$

(vi) The embedding of $\left(D\left[\mathcal{E}_{G}\right], \sqrt{\mathcal{E}_{G}+\delta_{o}^{2}}\right)$ into $C(X)$ is compact, that is, for a sequence of functions in $D\left[\mathcal{E}_{G}\right]$ which are uniformly bounded and whose energies are also uniformly bounded, there exists a subsequence that converges uniformly to a continuous function.

(vii) The form $\mathcal{E}_{G}$ is strong local, i.e., for $u, v \in D\left[\mathcal{E}_{G}\right], \mathcal{E}_{G}(u, v)=0$ if $u$ is constant on the support of $v$.

Now we are given a closed subset $K$ of $G$. For any $u \in C(K)$, let $\mathcal{A}_{u}=\left\{v \in D\left[\mathcal{E}_{G}\right] \mid v=u\right.$ on $\left.K\right\}$ and also $\mathcal{F}_{K}=\left\{u \in C(K) \mid \mathcal{A}_{u} \neq \emptyset\right\}$. Then in view of the properties (iii) and (iv), we see that for any $u \in \mathcal{F}_{K}$, there exists uniquely a minimizer $H_{K ; u}$ on $\mathcal{A}_{u}$, which is characterized as a function $h \in \mathcal{A}_{u}$ that satisfies $\mathscr{E}_{G}(h, v)=0$ for all $v \in D\left[\varepsilon_{G}\right]$ vanishing on $K$. Define a form $\varepsilon_{K}^{*}$ on $C(K)$ by

$$
D\left[\mathcal{E}_{K}^{*}\right]=\mathcal{F}_{K} ; \mathcal{E}_{K}^{*}(u, u)=\mathcal{E}_{G}\left(H_{K ; u}, H_{K ; u}\right), \quad u \in D\left[\mathcal{E}_{K}^{*}\right] .
$$

Then $\mathcal{E}_{K}^{*}$ satisfies the same properties of (i) through (vi) as $\mathcal{E}_{G}$; however (vii) does not hold true in general. We note that given a closed subset $L$ of $K$ and a function $u \in D\left[\mathcal{E}_{K}^{*}\right], \mathcal{E}_{K}^{*}(u, v)=0$ for all $v \in D\left[\mathcal{E}_{K}^{*}\right]$ with supp $v \subset K \backslash L$ if and only if $\mathcal{E}_{G}\left(H_{K ; u}, v\right)=0$ for all $v \in D\left[\mathcal{E}_{G}\right]$ with supp $v \subset G \backslash L$. In addition, let

$$
R_{K}^{*}(x, y)=\sup \left\{\frac{|u(x)-u(y)|^{2}}{\mathcal{E}_{K}^{*}(u, u)} \mid u \in D\left[\mathcal{E}_{K}^{*}\right], \mathcal{E}_{K}^{*}(u, u) \neq 0\right\}, \quad x, y \in K .
$$

Then $R_{K}^{*}$ is nothing but the restriction of the effective resistance $R_{G}$ of $G$ to the set $K$, i.e.,

$$
R_{K}^{*}(x, y)=R_{G}(x, y), \quad x, y \in K .
$$

Now the Markov property (v) implies that the maximum principle holds true for $H_{K ; u}$, that is,

$$
\min _{K} u \leq H_{K ; u} \leq \max _{K} u .
$$

Here we recall another important consequence from the Markov property that for $u, v \in D\left[\varepsilon_{K}^{*}\right], u v \in D\left[\varepsilon_{K}^{*}\right]$ and we have 


$$
\sqrt{\mathcal{E}_{K}^{*}(u v, u v)} \leq\|u\|_{C^{0}} \sqrt{\mathcal{E}_{K}^{*}(v, v)}+\|v\|_{C^{0}} \sqrt{\mathcal{E}_{K}^{*}(u, u)}
$$

(cf. [9], Theorem 1.4.2). In particular, $D\left[\mathcal{E}_{K}^{*}\right]$ is a subalgebra of $C(K)$ containing the unit element 1 and separating points of $K$. As a result, $D\left[\mathcal{E}_{K}^{*}\right]$ is dense in $C(K)$, since $K$ is compact.

Now we consider the case where $K$ consists of $N$ points $x_{1}, \ldots, x_{N}$ of $G$. We denote by $u_{i}(i=1, \ldots, N)$ the function on $K$ defined by $u_{i}\left(x_{j}\right)=\delta_{i j}$. Let $c_{i j}=\mathcal{E}_{K}^{*}\left(u_{i}, u_{j}\right)(i, j=1, \ldots, N)$. Since $\mathcal{E}_{K}^{*}\left(u_{i}, 1\right)=0$, we get $\sum_{j=1}^{N} c_{i j}=0(i=$ $1, \ldots, N)$, and hence we can deduce that

$$
\mathcal{E}_{K}^{*}(u, v)=-\frac{1}{2} \sum_{i, j=1}^{N} c_{i j}\left(u\left(x_{i}\right)-u\left(x_{j}\right)\right)\left(v\left(x_{i}\right)-v\left(x_{j}\right)\right), \quad u, v \in D\left[\mathcal{E}_{K}^{*}\right] .
$$

Note that $c_{i j} \leq 0$ if $i \neq j$, which follows from the Markov property of $\mathcal{E}_{K}^{*}(c f$. [20], p. 43).

\section{Green Functions, Effective Resistance, and Laplace Operators}

2.1 Let $G$ be a compact connected metric graph corresponding to a finite network $(V, E, r)$. For points $a, x \in G$, in view of (1), we have uniquely a function $g_{a}(x, *) \in D\left[\mathcal{E}_{G}\right]$ satisfying $\mathcal{E}_{G}\left(g_{a}(x, *), v\right)=v(x)-v(a)$ for all $v \in D\left[\mathcal{E}_{G}\right]$, and $g_{a}(x, a)=0$. Observe that $g_{a}(x, y)=g_{a}(y, x)$, since $g_{a}(x, y)=\varepsilon_{G}\left(g_{a}(x, *), g_{a}(y, *)\right)=\varepsilon_{G}\left(g_{a}(y, *), g_{a}(x, *)\right)=g_{a}(y, x)$. The functions $g_{a}$ are called the Green functions of the form $\varepsilon_{G}$. Given two points $x, y \in G$, let $H_{x y}(z)=g_{y}(z, x) / g_{y}(x, x)$ $(z \in G)$. Then the effective resistance $R_{G}$ is expressed as

$$
R_{G}(x, y)=\varepsilon_{G}\left(H_{x y}, H_{x y}\right)^{-1}=g_{y}(x, x)\left(=g_{x}(y, y)\right), \quad x, y \in G .
$$

In fact, we can show the following result, which will be verified after Theorem 2.2.

Theorem 2.1. The following mutually equivalent identities hold:

$$
\begin{gathered}
g_{z}(x, y)=\frac{1}{2}\left\{R_{G}(x, z)+R_{G}(z, y)-R_{G}(x, y)\right\}, \\
R_{G}(x, y)=g_{x}(y, y)=g_{y}(x, x)=g_{x}(y, z)+g_{y}(x, z)
\end{gathered}
$$

for all $x, y, z \in G$.

Since $g_{z}(x, y) \geq 0$ by the maximum principle, the first identity implies that $R_{G}: G \times G \rightarrow \mathbf{R}$ satisfies the triangle inequality and hence we have the following

Corollary 2.2. The effective resistance $R_{G}$ is a metric on $G$, called the resistance metric of $G$, which induces the same topology as the Riemannian distance.

Remarks (i) Let $G$ and $(V, E, r)$ be as above. Then the resistance metric of $G$ coincides with its Riemannian distance if and only if $G$ is simply connected. Let $\left(V^{\prime}, E^{\prime}\right)$ be a subnetwork of $(V, E)$, i.e., a pair of sets $V^{\prime} \subset V$ and $E^{\prime} \subset E$ such that $x, y \in V^{\prime}$ if $\{x, y\} \in E^{\prime}$. Let $G^{\prime}$ be the metric graph associated with the subnetwork $\left(V^{\prime}, E^{\prime}, r_{\left[E^{\prime}\right.}\right)$ and assume that $G^{\prime}$ is connected. Then we have

$$
R_{G}(x, y) \leq R_{G^{\prime}}(x, y), \quad x, y \in G^{\prime} .
$$

This is known as Rayleigh's monotonicity principle.

(ii) The identities in Theorem 2.1 are presented for finite networks in [27].

2.2 Let $\mu$ be a Radon measure on $G$ with support $K$ and consider the Laplace operator $\mathscr{L}_{\mu}$ associated with the closed form $\mathcal{E}_{K}^{*}$ on $L^{2}(K, \mu)$. The domain $D\left[\mathcal{L}_{\mu}\right]$ of $\mathcal{L}_{\mu}$ consists of functions $u \in D\left[\mathcal{E}_{K}^{*}\right]$ such that the functional on $D\left[\mathcal{E}_{K}^{*}\right]$ defined by $v \rightarrow \mathcal{E}_{K}^{*}(u, v)$ is continuous with respect to the norm of $L^{2}(K, \mu)$, and for any $u \in D\left[\mathcal{L}_{\mu}\right], \mathscr{L}_{\mu} u$ is the unique function (in the closure of $D\left[\mathcal{E}_{K}^{*}\right]$ in $L^{2}(K, \mu)$ ) satisfying

$$
\mathcal{E}_{K}^{*}(v, u)=\int_{K} v \mathscr{L}_{\mu} u d \mu, \quad v \in D\left[\mathcal{E}_{K}^{*}\right] .
$$

Let

$$
g^{\mu}(x, y)=\frac{1}{\mu(K)} \int_{K} g_{z}(x, y) d \mu(z), \quad x, y \in K .
$$

Then by the definition of the Green functions, we have

$$
u(x)=\frac{1}{\mu(K)} \int_{K} u d \mu+\int_{K} g^{\mu}(x, y) \mathcal{L}_{\mu} u(y) d \mu(y), \quad u \in D\left[\mathcal{L}_{\mu}\right], x \in K,
$$

and hence 


$$
u(x)=\frac{1}{\mu(K)} \int_{K} u d \mu+\mathcal{E}_{K}^{*}\left(g^{\mu}(x, *), u\right), \quad u \in D\left[\mathcal{E}_{K}^{*}\right], x \in K .
$$

Applying this to the functions $g_{z}(x, *)$, we can verify the following identity:

$$
g_{z}(x, y)=g^{\mu}(z, z)-g^{\mu}(z, x)-g^{\mu}(z, y)+g^{\mu}(x, y), \quad x, y, z \in K .
$$

Since $R_{G}(x, y)=g_{x}(y, y)$, direct computations show the following

Theorem 2.3. The effective resistance on $K$ is given by

$$
R_{G}(x, y)=g^{\mu}(x, x)-2 g^{\mu}(x, y)+g^{\mu}(y, y), \quad x, y \in K .
$$

Now by taking a Radon measure $\mu$ such that $\operatorname{supp} \mu=G$ and using (2), we can derive the first identity of Theorem 2.1, from which the remaining one follows.

Let

$$
g_{*}^{\mu}(x, y):=g^{\mu}(x, y)-C_{\mu}, \quad x, y \in K,
$$

and

$$
g_{\mu} u(x)=\int_{K} g_{*}^{\mu}(x, y) u(y) d \mu(y), \quad u \in L^{2}(K, \mu),
$$

where $C_{\mu}$ is a positive constant given by

$$
C_{\mu}=\frac{1}{\mu(K)} \int_{K} g_{\mu}(x, y) d \mu(y)=\frac{1}{2 \mu(K)^{2}} \int_{K} \int_{K} R_{G}(y, z) d \mu(y) d \mu(z) .
$$

Then $\mathcal{g}_{\mu}$ turns out to be the Green operator of $\mathcal{L}_{\mu}$, that is, it satisfies

$$
\begin{aligned}
I=\mathscr{H}_{\mu}+\mathcal{L}_{\mu} \mathcal{g}_{\mu} & \text { on } L^{2}(K, \mu), \\
I & =\mathscr{H}_{\mu}+\mathcal{g}_{\mu} \mathcal{L}_{\mu} \quad \text { on } D\left[\mathcal{L}_{\mu}\right], \\
\mathscr{H}_{\mu} \mathcal{g}_{\mu} & =\mathcal{g}_{\mu} \mathcal{H}_{\mu}=0,
\end{aligned}
$$

where we put

$$
\mathscr{H}_{\mu} u=\frac{1}{\mu(K)} \int_{K} u d \mu .
$$

Let $N=\# K \leq+\infty$ and $\lambda_{i}(i=0,1, \ldots, N-1)$ the $i$-th eigenvalue of $\mathcal{L}_{\mu}$, where $\lambda_{0}=0$. We take a complete orthonormal system of eigenfunctions $\phi_{i}$ with eigenvalue $\lambda_{i}$ in $L^{2}(K, \mu)$. Then the Green kernel $g_{*}^{\mu}$ is given by

$$
g_{*}^{\mu}(x, y)=\sum_{i=1}^{N-1} \frac{1}{\lambda_{i}} \phi_{i}(x) \phi_{i}(y) .
$$

Since $R_{G}(x, y)=g_{*}^{\mu}(x, x)-2 g_{*}^{\mu}(x, y)+g_{*}^{\mu}(y, y)$, as a result of Theorem 2.3, we obtain

Corollary 2.4. It holds:

$$
R_{G}(x, y)=\sum_{i=1}^{N-1} \frac{1}{\lambda_{i}}\left(\phi_{i}(x)-\phi_{i}(y)\right)^{2}, \quad x, y \in K .
$$

In particular, one has

$$
\int_{K} \int_{K} R_{G}(x, y) d \mu(x) d \mu(y)=2 \mu(K) \sum_{i=1}^{N-1} \frac{1}{\lambda_{i}} .
$$

From the last identity of the corollary, it is easy to see that

$$
\lambda_{i} \geq \frac{2 i}{D\left(K, R_{G}\right) \mu(K)}, \quad i=1,2, \ldots,
$$

where $D\left(K, R_{G}\right)=\sup \left\{R_{G}(x, y) \mid x, y \in K\right\}$.

2.3 Now we consider the case where a closed subset $K$ of a compact metric graph $G$ consists of a finite number of points $\left\{x_{1}, \ldots, x_{N}\right\}(N<+\infty)$. We define $N$ functions $u_{i}(i=1, \ldots, N)$ on $K$ by $u_{i}\left(x_{j}\right)=\delta_{i j}$. Then $u_{i} \in D\left[\mathcal{E}_{K}^{*}\right]$. Let $c_{i j}=\varepsilon_{K}^{*}\left(u_{i}, u_{j}\right)$. For every fixed $k, 1 \leq k \leq N$, the definition of the Green functions reads 


$$
\sum_{\ell=1}^{N} g_{x_{k}}\left(x_{i}, x_{\ell}\right) c_{\ell j}=\delta_{i j}, \quad i \neq k, j \neq k,
$$

that is, defining $(N-1) \times(N-1)$ matrices $C_{k}$ and $G_{k}$ respectively by $C_{k}=\left(c_{i j}\right)$ and $G_{k}=\left(g_{x_{k}}\left(x_{i}, x_{j}\right)\right)$, where $i \neq k, j \neq k$, we have

$$
G_{k} C_{k}=I_{N-1}, \quad k=1, \ldots, N,
$$

where $I_{N-1}$ stands for the unit matrix. By (4), we get

$$
\sum_{k=1}^{N} \sum_{i, j=1}^{N} g_{x_{k}}\left(x_{i}, x_{j}\right) c_{i j}=N(N-1) .
$$

Since $g_{x_{k}}\left(x_{i}, x_{j}\right)=\frac{1}{2}\left(R_{G}\left(x_{i}, x_{k}\right)+R_{G}\left(x_{k}, x_{j}\right)-R_{G}\left(x_{i}, x_{j}\right)\right)$ and $\sum_{j=1}^{N} c_{i j}=0$, we thus obtain the following:

$$
-\frac{1}{2} \sum_{i, j=1}^{N} R_{G}\left(x_{i}, x_{j}\right) \mathcal{E}_{K}^{*}\left(u_{i}, u_{j}\right)=N-1
$$

When $K$ is the set of all vertecies $V, c_{i j}=-1 / r(i, j)(i \neq j)$, and hence the identity reads

$$
\frac{1}{2} \sum_{i, j=1, i \neq j}^{N} \frac{R_{G}\left(x_{i}, x_{j}\right)}{r(i, j)}=N-1 .
$$

This is a classical result due to R. M. Foster ( $c f$. [7, 27]).

Now taking a canonical measure on $K$ defined by $\mu^{c}=\sum_{i=1}^{N} \delta_{x_{i}}$ and using the identities of Corollary 2.4, we have the following

Corollary 2.5. Under the conditions as above, one has

$$
\max _{1 \leq k \leq n-1} \frac{2 k}{(n-1) \lambda_{k}\left(\mu^{c}\right)} \leq \max _{x_{i}, x_{j} \in K} R_{G}\left(x_{i}, x_{j}\right) \leq \frac{2}{\lambda_{1}\left(\mu^{c}\right)}
$$

and

$$
\frac{2}{\lambda_{n-1}\left(\mu^{c}\right)} \leq R_{G}\left(x_{i}, x_{j}\right), \quad x_{i}, x_{j} \in K, x_{i} \neq x_{j}
$$

Remark The second inequality in this corollary is proved in [2].

2.4 Let $V$ be a finite set, $V=\left\{x_{1}, \ldots, x_{N}\right\}$, and consider a nonnegative quadratic form $\varepsilon$ on the space $\ell(V)$ of functions on $V$ such that $\mathcal{E}(u, u)=0$ if and only if $u$ is constant. Let $R_{\mathcal{E}}(x, y)=\max \left\{|u(x)-u(y)|^{2} / \mathscr{E}(u, u) \mid u \in \ell(V), \mathcal{E}(u, u)>\right.$ $0\}(x, y \in V)$. Then the arguments and the results as in the previous subsections 2.1 through 2.3 are valid, except the nonnegativity of the Green functions $g_{z}(x, y)$ of $\&$; this relies on the maximum principle under the Markov property. In fact, if we assume that the form satisfies the Markov property, then we see that $c_{i j}=\mathcal{E}\left(u_{i}, u_{j}\right) \leq 0$ for all $i, j=1, \ldots, N$ with $i \neq j$, where $\left\{u_{1}, \ldots, u_{N}\right\}$ is a canonical basis of $\ell(V)$ described in subsection 2.3 . In this case, by letting $E=$ $\left\{\{x, y\} \subset V \mid c_{i j}<0\right\}$ and $r(i, j)=-1 / c_{i j}$, we obtain a network $(V, E, r)$. In particular, Theorem 2.1 holds true without the Markov property, and hence we have the following

Theorem 2.6. Let $V$ be a finite set. Let $\varepsilon_{\alpha}(\alpha=1,2)$ be nonnegative quadratic forms on $\ell(V)$ such that $\varepsilon_{\alpha}(u, u)=0$ if and only if $u$ is constant. Then $\mathcal{E}_{1}=\mathcal{E}_{2}$ if and only if $R_{\mathcal{E}_{1}}=R_{\mathcal{E}_{2}}$.

Given a subset $K$ of $V$, we denote as before by $\mathcal{E}_{K}^{*}$ a form on $\ell(K)$ defined by $\mathcal{E}_{K}^{*}(u, u)=\inf \{\mathscr{E}(v, v) \mid v \in \ell(V)$, $\left.v_{\mid K}=u\right\}$. Then we have $R_{\mathcal{E}_{K}^{*}}(x, y)=R_{\mathcal{E}}(x, y)$ for all $x, y \in K$. As a result of Theorem 2.6, we have the following

Corollary 2.7. Let $K$ be a subset of a finite set $V$. Let $\mathcal{E}$ and $\mathcal{F}$ be respectively quadratic forms on $\ell(V)$ and $\ell(K)$ as above. Then $\mathcal{F}=\mathcal{E}_{K}^{*}$ if and only if $R_{\mathcal{F}}(x, y)=R_{\mathcal{E}}(x, y)$ for all $x, y \in K$.

Theorem 2.6 and Corollary 2.7 are respectively proved in [20], Theorem 2.1.12 and Corollary 2.1.13, under the condition of the Markov property, and they affermatively answer an question raised there.

2.5 In this part, we recall some definitions and results on resistance forms and resistance metrics from [20], Chap. 2.

Let $X$ be a set and $(\mathcal{E}, D[\mathcal{E}]$ ) a pair of a linear subspace $D[\mathcal{E}]$ of the space $\ell(X)$ of all functions on $X$ and a nonnegative quadratic form $\mathcal{E}$ on $D[\mathcal{E}$. We call such a pair $(\mathcal{E}, D[\mathcal{E}])$ a resistance form on $X$ if it satisfies the following conditions (RF-i) through $(\mathrm{RF}-\mathrm{v})$ :

(RF-i) $\mathcal{E}(u, u)=0$ if and only if $u$ is constant on $X$.

(RF-ii) Let $\sim$ be an equivalence relation on $D[\mathcal{E}]$ defined by $u \sim v$ if and only if $u-v$ is constant on $X$. Then $(D[\mathcal{E}] / \sim, \mathcal{E})$ is a Hilbert space. 
(RF-iii) For any finite subset $V$ of $X$ and for any $u \in \ell(V)$, there exists $v \in D[\mathcal{E}]$ such that $\left.v\right|_{V}=u$.

(RF-iv) For any $x, y \in X, \sup \left\{|u(x)-u(y)|^{2} / \mathcal{E}(u, u) \mid u \in D[\mathcal{E}], \mathcal{E}(u, u)>0\right\}$ is finite. The supremum is denoted by $R_{\mathcal{E}}(x, y)$, which will be called the effective resistance between the points $x$ and $y$.

(RF-v) If $u \in D[\mathcal{E}]$, then $\bar{u} \in D[\mathcal{E}]$ and $\mathcal{E}(\bar{u}, \bar{u}) \leq \mathcal{E}(u, u)$, where $\bar{u}$ is defined by $\bar{u}(x)=1$ if $u(x) \geq 1, \bar{u}(x)=u(x)$ if $0<u(x)<1$, and $\bar{u}(x)=0$ if $u(x) \leq 0$.

We use $\mathcal{R} \mathcal{F}(X)$ to denote the collection of resistance forms on $X$. Given $(\mathcal{E}, D[\mathcal{E}]) \in \mathcal{R} \mathcal{F}(X)$ and a finite subset $V$ of $X$, let

$$
\mathcal{E}_{V}^{*}(u, u)=\inf \left\{\mathcal{E}(v, v) \mid v \in D[\mathcal{E}], v_{\mid V}=u\right\}, \quad u \in \ell(V) .
$$

Then $\left(\mathcal{E}_{V}^{*}, \ell(V)\right) \in \mathcal{R} \mathcal{F}(V)$ and $R_{\mathcal{E}_{V}^{*}}=R_{\mathcal{E}}$ on $V$. This implies that if $(\mathcal{E}, D[\mathcal{E}]) \in \mathcal{R} \mathcal{F}(X)$, then $R_{\mathcal{E}}$ induces a metric on $X$, called the resistance metric associated with the resistance form $\mathcal{E}$ on $X$. Obviously $\sqrt{R_{\mathcal{E}}}$ is also a distance on $X$; indeed, so is it without the Markov property (RF-v), if we assume instead of property $\left(\operatorname{RF}\right.$-iii) that $R_{\mathcal{E}}(x, y)>0$ for all $x, y \in X$ with $x \neq y$.

A function $R: X \times X \rightarrow[0,+\infty)$ is by definition a resistance metric on $X$ if, for any finite subset $V \subset X$, there exists a resistance form $\mathcal{E}_{V}$ on $\ell(V)$ such that $R(x, y)=R_{\mathcal{E}_{V}}(x, y)$ for all $x, y \in V$, where $R_{\mathcal{E}_{V}}$ is the effective resistance with respect to the form $\mathcal{E}_{V}$. The collection of resistance metrics on $X$ is denoted by $\mathcal{R} \mathcal{M}(X)$. Each $(\mathcal{E}, D[\mathcal{E}]) \in \mathcal{R} \mathcal{F}(X)$ is associated with $R_{\mathcal{E}} \in \mathcal{R} \mathcal{M}(X)$. This correspondence is in fact bijective.

We note that, when $\left(X, \sqrt{R_{\mathcal{E}}}\right)$ is separable, $\mathcal{E}$ can be thought to be a limit of finite dimensional forms; in fact, given an increasing sequence $\left\{V_{m}\right\}$ of finite subsets of $X$ such that $\cup_{m} V_{m}$ is dense in $X$, a function $u$ on $X$ belongs to $D[\mathcal{E}]$ if and only if $\lim _{m \rightarrow \infty} \mathcal{E}_{V_{m}}^{*}\left(u_{\mid V_{m}}, u_{\mid V_{m}}\right)<+\infty$ and in this case, $\mathcal{E}(u, u)=\lim _{m \rightarrow \infty} \mathcal{E}_{V_{m}}^{*}\left(u_{\mid V_{m}}, u_{\mid V_{m}}\right)$, where $\mathcal{E}_{V_{m}}^{*}$ is the induced form on $\ell\left(V_{m}\right)$ as above.

Given $(\mathcal{E}, D[\mathcal{E}]) \in \mathcal{R} \mathcal{F}(X)$, if $\bar{X}$ is the completion of $X$ with respect to the resistance metric, then any $u \in D[\mathcal{E}]$ is naturally extended to a continuous function on $\bar{X}$. Using this extention, $\mathcal{E}$ is regarded as the collection of functions on $\bar{X}$. Then $(\mathcal{E}, D[\mathcal{E}])$ is a resistance form on $\bar{X}$ and the resistance metric associated with $(\mathcal{E}, D[\mathcal{E}])$ on $\bar{X}$ is the natural extension of the resistance metric associated with $(\mathcal{E}, D[\mathcal{E}])$ on $X$.

2.6 Before proceeding to the next section, we consider a wider class of quadratic forms for our purpose. Let $(\&, D[\&])$ be a nonnegative quadratic form on a set $X$ and

$$
R_{\mathcal{E}}(x, y)=\sup \left\{\frac{|u(x)-u(y)|^{2}}{\mathcal{E}(u, u)} \mid u \in D[\mathcal{E}], \mathcal{E}(u, u)>0\right\}, \quad x, y \in X .
$$

We suppose that $0<R_{\mathcal{E}}(x, y)<+\infty$ for all $x, y \in X$ with $x \neq y$. Then $\sqrt{R_{\mathcal{E}}}$ induces a distance on $X$, and the domain $D[\mathcal{E}]$ is a subspace of $C\left(X, \sqrt{R_{\mathcal{E}}}\right)$. Moreover we suppose that for a point $o \in X$, a quadratic form defined by $\mathcal{E}(u, v)+$ $u(o) v(o)$ provides a complete inner product on $D[\mathcal{E}]$, that is, $\left(D[\mathcal{E}], \mathcal{E}+\delta_{o}^{2}\right)$ is a Hilbert space (this holds for any $o \in X$ because of the assumption above).

Now we suppose that the metric space $\left(X, \sqrt{R_{\mathcal{E}}}\right)$ is separable. Then we are able to verify the following assertions:

(i) When the form $\mathcal{E}$ is regarded as a functional on the space of continuous functions $C(X)$ on $X=\left(X, \sqrt{R_{\mathcal{E}}}\right)$ by letting $\mathscr{E}(u)=\mathscr{E}(u, u)$ if $u \in D[\mathcal{E}]$ and $\mathscr{E}(u)=+\infty$ otherwise, the functional $\mathscr{E}: C(X) \rightarrow[0,+\infty]$ is lower semicontinuous with respect to the uniform topology of $C\left(X, \sqrt{R_{\mathcal{E}}}\right)$.

(ii) Given a closed subset $K$ of $X$, let

$$
\begin{aligned}
& D\left[\mathcal{E}_{K}^{*}\right]=\left\{u \in C(K) \mid u=v_{\mid K} \exists v \in D[\mathcal{E}]\right\}, \\
& \mathcal{E}_{K}^{*}(u, u)=\inf \left\{\mathcal{E}(v, v) \mid v \in D[\mathcal{E}], v_{\mid K}=u\right\} .
\end{aligned}
$$

Then $\left(\varepsilon_{K}^{*}, D\left[\mathcal{E}_{K}^{*}\right]\right)$ is a quadratic form on $C(K)$ with the same properties as $\mathscr{E}$ on $X$. In fact, there exists a unique minimizer $H_{K ; u}$ and the correspondence $u \in D\left[\mathcal{E}_{K}^{*}\right] \rightarrow H_{K ; u} \in D[\mathcal{E}]$ is linear and injective. The minimizer is characterized as a function $h \in D[\mathcal{E}]$ which coincides with $u$ on $K$ and satisfies $\mathscr{E}(h, v)=0$ for all $v \in D[\mathcal{E}]$ vanishing on $K$. In addition, we see that $\sup \left\{|u(x)-u(y)|^{2} / \mathcal{E}_{K}^{*}(u, u) \mid u \in D\left[\mathcal{E}_{K}^{*}\right], \mathcal{E}_{K}^{*}(u, u) \neq 0\right\}=R(x, y)$ for all $x, y \in K$.

(iii) If $1 \in D[\mathcal{E}]$ and $\mathcal{E}(1,1)=0$, then Theorem 2.1 is valid for this situation. If, in addition, $\mathcal{E}$ satisfies the Markov property, then so does $\varepsilon_{K}^{*}$, and hence $D\left[\mathcal{E}_{K}^{*}\right] \cap L^{\infty}$ is a subalgebra of $C(K)$ containing the unit elememt 1 and separating points of $K$; thus in this case, $(\mathcal{E}, D[\mathcal{E}])$ is a resistance form on $X$.

(iv) Let $\mu$ be a $\sigma$-finite Borel measure on $X$ and $K$ the support of $\mu$. Let $H$ be the $L^{2}$-closure of $D\left[\mathcal{E}_{K}^{*}\right] \cap L^{2}(K, \mu)$ and $\mathcal{L}_{\mu}$ the self-adjoint operator associated with the closed form $\mathcal{E}_{K}^{*}$ in $H$ with domain $D\left[\mathcal{E}_{K}^{*}\right] \cap L^{2}(K, \mu)$. Given a positive number $\alpha$, we use $\mathcal{R}_{\mu ; \alpha}$ to denote the resolvent operator $\left(\mathcal{L}_{\mu}+\alpha I\right)^{-1}$ of $\mathcal{L}_{\mu}$. Suppose that

$$
\mu(K)<+\infty, \int_{K} R_{\mathcal{E}}(o, x) d \mu(x)<+\infty
$$

for some $o \in X$ (and hence any $o$ ). Then $D\left[\mathcal{E}_{K}^{*}\right] \subset L^{2}(K, \mu)$ and the embedding is compact with respect to the norm $\left(\mathcal{E}_{K}^{*}(u, u)+\int u^{2} d \mu\right)^{1 / 2}$ of $D\left[\mathcal{E}_{K}^{*}\right]$. If, in addition, $1 \in D[\mathcal{E}]$ and $\mathcal{E}(1,1)=0$, then Theorem 2.3 , Corollary 2.4 and Corollary 2.5 hold true. 
Example Let $V$ be a set of $N$ points, $V=\left\{x_{1}, \ldots, x_{N}\right\}$, and $\mu^{c}=\sum_{i=1}^{N} \delta_{x_{i}}$. Let $\left\{e_{0}, e_{1}, \ldots, e_{N-1}\right\}$ be an orthonormal basis of $L^{2}\left(V, \mu^{c}\right)(=\ell(V))$ such that $e_{0}(x)=1 / \sqrt{N}$ for all $x \in V$. Given $k, 0<k<N$, and positive numbers $\lambda_{i}$ $(i=1, \ldots, k)$ such that $\lambda_{1} \leq \cdots \leq \lambda_{k}$, we define a quadratic form $(\mathcal{E}, D[\mathcal{E}])$ on $V$ by $D[\mathcal{E}]=\left\{u=\sum_{i=1}^{k} u_{i} e_{i} \mid u \in \ell(V)\right\}$ and $\mathcal{E}(u, v)=\sum_{i=1}^{k} \lambda_{i} u_{i} v_{i}, u, v \in D[\mathcal{E}]$. Then the effective resistance $R_{\mathcal{E}}$ is given by

$$
R_{\mathcal{E}}(x, y)=\sum_{i=1}^{k} \frac{1}{\lambda_{i}}\left(e_{i}(x)-e_{i}(y)\right)^{2}, \quad x, y \in V .
$$

We observe that if, for any pair of points $x, y \in V$, there exists an $e_{i}, 1 \leq i \leq k$, such that $e_{i}(x) \neq e_{i}(y)$, that is, $\left\{e_{1}, \ldots, e_{k}\right\}$ separates the points of $V$, then $\sqrt{R_{\mathcal{E}}}$ is a distance on $V$. We also note that given a sequence of positive numbers $\varepsilon_{n}$ tending to 0 as $n \rightarrow \infty$, a sequence of the forms $\mathcal{E}_{n}$ defined by $\mathcal{E}_{n}(u, u)=\sum_{i=1}^{k} \lambda_{i} u_{i}^{2}+\varepsilon_{n}^{-1} \sum_{j=k+1}^{N-1} u_{j}^{2}$ converges to $(\mathcal{E}, D[\mathcal{E}])$ as $n \rightarrow \infty$.

Consider the case where $N=3, k=1, \lambda_{1}=1$ and $e_{1}=\left(2\left(\alpha^{2}+\alpha+1\right)\right)^{-1 / 2}(1, \alpha,-1-\alpha)$. Then the Green functions of $\mathcal{E}$ are all nonnegative if and only if $-2<\alpha<-1 / 2$; in this case, $R_{\mathcal{E}}$ satisfies the triangle inequality.

\section{Gromov-Hausdorff and Variational Convergence}

3.1 Let us recall first the definition of the Gromov-Hausdorff convergence of metric spaces. Let $X$ and $Y$ be metric spaces and $\varepsilon>0$. A (not necessarily continuous) map $f: X \rightarrow Y$ is called an $\varepsilon$-Hausdorff approximation if

$$
\sup _{x_{1}, x_{2} \in X}\left|d_{Y}\left(f\left(x_{1}\right), f\left(x_{2}\right)\right)-d_{X}\left(x_{1}, x_{2}\right)\right|<\varepsilon
$$

and the image $f(X)$ of $f$ is an $\varepsilon$-net in $Y$, i.e., $d_{Y}(y, f(X))<\varepsilon$ for every $y \in Y$. By definition, a sequence of compact metric spaces $\left\{X_{n}\right\}$ converges to a compact metric space $X$ in the Gromov-Hausdorff sense if there exist $\varepsilon_{n}$-Hausdorff approximations $f_{n}: X_{n} \rightarrow X$ with $\lim _{n \rightarrow \infty} \varepsilon_{n}=0$.

We remark that every compact length space can be obtained as a Gromov-Hausdorff limit of compact metric graphs endowed with their Riemannian distances ( $c f$. [1], Proposition 7.5.5).

A pointed metric space is a pair $(X, p)$ of a metric space $X$ and a point $p \in X$. A sequence $\left\{\left(X_{n}, p_{n}\right)\right\}$ of pointed metric spaces converges to a pointed metric space $(X, p)$ in the Gromov-Hausdorff sense if the following holds. For every $r>0$ and $\varepsilon>0$, there exists a positive integer $n_{0}$ such that for any $n>n_{0}$, there is a map $f$ from the ball $B_{r}\left(p_{n}\right)$ around $p_{n}$ with radius $r$ in $X_{n}$ to $X$ satisfying the following properties:

(i) $f\left(p_{n}\right)=p$;

(ii) $\sup \left\{\left|d_{X}\left(f\left(x_{1}\right), f\left(x_{2}\right)\right)-d_{X_{n}}\left(x_{1}, x_{2}\right)\right| \mid x_{1}, x_{2} \in B_{r}\left(p_{n}\right)\right\}<\varepsilon$;

(iii) the $\varepsilon$-neighborhood of the set $f\left(B_{r}\left(p_{n}\right)\right)$ contains the ball $B_{r-\varepsilon}(p)$ centered at $p$ of radius $r-\varepsilon$.

Now let us consider a set of finite networks.

Lemma 3.1. Let $\left\{\Gamma_{n}=\left(V, E_{n}, r_{n}\right)\right\}$ be a sequence of finite networks of the same set of verticies $V$. Then the resistance metric $R_{n}$ of $\Gamma_{n}$ converges to a metric $R$ on $V$ as $n \rightarrow \infty$, that is, $\lim _{n \rightarrow \infty} R_{n}(x, y)=R(x, y)$ for all $x, y \in V$, if and only if the resistance form $\mathcal{E}_{n}$ of $\Gamma_{n}$ converges to a resistance form $\mathcal{E}$ on $\ell(V)$ as $n \rightarrow \infty$ in the sense that

(i) for any sequence of functions $u_{n}$ on $V$ converging to a function $u$ on $V$, we have

$$
\mathcal{E}(u, u) \leq \liminf _{n \rightarrow \infty} \mathcal{E}_{n}\left(u_{n}, u_{n}\right)(\leq+\infty)
$$

(ii) for every function $u$ on $V$, there exists a sequence of functions $u_{n}$ converging to the function $u$ such that

$$
\limsup _{n \rightarrow \infty} \mathcal{E}_{n}\left(u_{n}, u_{n}\right) \leq \mathcal{E}(u, u)(\leqq+\infty)
$$

Now using this lemma, we can show the following

Theorem 3.2. Let $\left\{\left(V_{n}, E_{n}, r_{n}, R_{n}\right)\right\}$ be a sequence of finite networks with the resistance metrics $R_{n}$ and $p_{n}$ a point of $V_{n}$. Suppose that a sequence of pointed metric spaces $\left(V_{n}, p_{n}, R_{n}\right)$ converges to a pointed metric space $(X, p, R)$ in the Gromov-Hausdorff sense. Then the distance $R$ is the resistance metric on $X$ associated with a resistance form $(\mathcal{E}, D[\mathcal{E}])$ on $X$. Moreover for any finite subset $V \subset X$, let $\left\{W_{n}\right\}$ be a sequence of finite subsets $W_{n}$ of $V_{n}$ such that \#W $W_{n}=\# V$, $W_{n} \subset B_{r}\left(p_{n}\right)$ for large $r$ and the image of $W_{n}$ by approximating maps $f_{n}: B_{r}\left(p_{n}\right) \rightarrow X$ converges to $V$ as $n \rightarrow \infty$. Then the induced form $\mathcal{E}_{W_{n}}^{*}$ on $\ell\left(W_{n}\right)$ converges to the induced form $\mathcal{E}_{V}^{*}$ on $\ell(V)$ in the same way as in Lemma 3.1.

Example Let $\Gamma=(V, E, r)$ be a locally finite, connected infinite network and $R_{\Gamma}$ the resistance metric on $V$. Let us consider an increasing sequence of finite subsets $V_{n}$ which exhausts $V$, that is, $V=\bigcup_{n} V_{n}$, and the subnetworks $\Gamma_{n}=\left(V_{n}, E_{n}, r_{n}\right)$. Fix a point $p \in V$. Then in view of Rayleigh's monotonicity principle, a sequence of pointed metric spaces $\left(V_{n}, p, R_{\Gamma_{n}}\right)$ with the resistance metrics $R_{\Gamma_{n}}$ converges to $\left(V, p, R_{\Gamma}\right)$ in the Gromov-Hausdorff sense as $n \rightarrow \infty$.

3.2 We are given a compact, separable, Hausdorff space $Y$ and a sequence of such spaces $X_{n}$, and also a sequence of maps $f_{n}: X_{n} \rightarrow Y$. We say that a sequence of functions $u_{n} \in C\left(X_{n}\right)$ uniformly converges to a function $u \in C(Y)$ (via $\left.f_{n}\right)$ 
if $\lim _{n \rightarrow \infty} \sup _{X_{n}}\left|u \circ f_{n}-u_{n}\right|=0$. Let $\mathcal{F}: C(Y) \rightarrow[0,+\infty]$ and $\mathcal{F}_{n}: C\left(X_{n}\right) \rightarrow[0,+\infty]$ be lower semi-continuous functionals on $C(Y)$ and $C\left(X_{n}\right)$ respectively. We say that $\mathcal{F}_{n} \Gamma$-converges to $\mathcal{F}$ if the following conditions are satisfied: (i) if a sequence of functions $u_{n} \in C\left(X_{n}\right)$ uniformly converges to a function $u \in C(Y)$, then we have

$$
\mathcal{F}(u) \leq \liminf _{n \rightarrow \infty} \mathcal{F}_{n}\left(u_{n}\right)(\leq+\infty) ;
$$

(ii) for any $u \in C(Y)$, there exists a sequence of functions $u_{n} \in C\left(X_{n}\right)$ such that $u_{n}$ uniformly converges to $u$ and

$$
\limsup _{n \rightarrow \infty} \mathcal{F}_{n}\left(u_{n}\right) \leq \mathcal{F}(u)(\leq+\infty) .
$$

The following is a basic fact on this variational convergence.

Theorem 3.3. Let $Y$ and $\left\{X_{n}\right\}$ be respectively a compact, separable, Hausdorff space and a sequence of such spaces. Given a sequence of maps $f_{n}: X_{n} \rightarrow Y$ and a sequence of lower semi-continuous functionals $\mathcal{F}_{n}: C\left(X_{n}\right) \rightarrow[0,+\infty]$, there exsists a subsequence, $\mathcal{F}_{m}$, and a lower semi-continuous functional $\mathcal{F}: C(Y) \rightarrow[0,+\infty]$ such that $\mathcal{F}_{m} \Gamma$ converges to $\mathcal{F}$ as $m \rightarrow \infty$.

Proof. Using the idea of De Giorgi's $\Gamma$-convergence (cf. e.g. [5],), we introduce a functional on $C(Y)$ as follows: Let $\mathscr{B}=\left\{O_{i}\right\}$ be a countable basis of $C(Y)$ such that $O_{i}$ is totally bounded. Given $O_{i}$ and a positive integer $k$, let

$$
O_{i, k ; n}=\left\{v \in C\left(X_{n}\right)\left|\sup _{X_{n}}\right| u \circ f_{n}-v \mid<1 / k \text { for some } u \in O_{i}\right\},
$$

and

$$
E_{i, k ; n}=\inf \left\{\mathcal{F}_{n}(v) \mid v \in O_{i, k ; n}\right\}(\leq+\infty) .
$$

Then passing to a subsequence, $\left\{X_{m}\right\}$, we may assume that for any $O_{i}$ and every $k, E_{i, k ; m}$ tends to an extended number $E_{i, k} \in[0,+\infty]$ as $m \rightarrow \infty$, and thus we are able to obtain a lower semi-continuous functional $\mathcal{F}: C(Y) \rightarrow[0,+\infty]$ defined by

$$
\mathscr{F}(u)=\sup \left\{E_{i, k} \mid u \in O_{i}, k>0\right\}, \quad u \in C(Y),
$$

to which $\mathcal{F}_{m} \Gamma$-converges as $m \rightarrow \infty$.

Let $\mathcal{F}_{n}: C\left(X_{n}\right) \rightarrow[0,+\infty]$ and $\mathcal{F}: C(Y) \rightarrow[0,+\infty]$ be as in Theorem 3.3. If $\mathcal{F}_{n}$ is induced from a quadratic form $\mathcal{E}_{n}$ on a subspace $D[\mathcal{E}]$ of $C\left(X_{n}\right)$, that is, $\mathcal{F}(u)=\mathcal{E}(u, u)$ for $u \in D[\mathcal{E}]$ and $\mathcal{F}(u)=+\infty$ for $u \in C\left(X_{n}\right) \backslash D[\mathcal{E}]$, then the $\Gamma$ limit $\mathcal{F}: C(Y) \rightarrow[0, \infty)$ is also induced from a quadratic form $\mathcal{E}$ on a subspace $D[\mathcal{E}]$ of $C(Y)$. For the functional induced from a quadratic frm $\mathscr{E}: D[\mathcal{E}] \times D[\mathcal{E}] \rightarrow \mathbf{R}$, we do not distinguish between the functional and the form $\mathcal{E}$. If all $\mathcal{F}_{n}$ satisfy the property that $\mathcal{F}_{n}(\bar{u}) \leq \mathcal{F}(u), u \in C\left(X_{n}\right)$, then so does the $\Gamma$-limit $\mathcal{F}$, where for a continuous function $u \in C\left(X_{n}\right)$, we set $\bar{u}=\max \{0, \min \{u, 1\}\}$.

3.3 Now we state some results. Let $\left\{\left(X_{n}, R_{n}\right)\right\}$ be a sequence of compact metric spaces of resistance forms $\mathcal{E}_{n}$. We assume that the following conditions are satisfied:

(i) There exist a compact, separable Hausdorff space $Y$, a sequence of maps $f_{n}: X_{n} \rightarrow Y$ and also a sequence of maps $h_{n}: Y \rightarrow X_{n}$ such that $f_{n} \circ h_{n}$ uniformly converges to the identity map of $Y$ as $n \rightarrow \infty$.

(ii) The functional $\varepsilon_{n} \Gamma$-converges (via $f_{n}$ ) to a functional $\varepsilon: C(Y) \rightarrow[0,+\infty]$ as $n \rightarrow \infty$.

(iii) For any sequence of functions $u_{n} \in D\left[\mathcal{E}_{n}\right]$ such that $\sup _{n} \max _{X_{n}}\left|u_{n}\right|<+\infty$ and $\sup _{n} \varepsilon_{n}\left(u_{n}, u_{n}\right)<+\infty$, there exists a subsequence $\left\{u_{m}\right\}$ which uniformly converges to a function $u \in C(Y)$ as $m \rightarrow \infty$.

Under these conditions, our main result is stated in

Theorem 3.4. The following assertions hold:

(iv) Let

$$
R_{\mathcal{E}}(x, y)=\sup \left\{\frac{|u(x)-u(y)|^{2}}{\mathcal{E}(u, u)} \mid u \in D[\mathcal{E}], \mathcal{E}(u, u) \neq 0\right\}, \quad x, y \in Y .
$$

Then $R_{\mathcal{E}}: Y \times Y \rightarrow[0,+\infty]$ induces a continuous pseudo-distance on $Y$ (admitting $+\infty$ in its values), and one has

$$
0 \leq R_{\mathcal{E}}(x, y)=\lim _{n \rightarrow \infty} R_{n}\left(h_{n}(x), h_{n}(y)\right) \leq+\infty, \quad x, y \in Y .
$$

(v) On $Y$, an equivalence relation $\sim_{b}$ is introduced as follows: $x \sim_{b} y$ if and only if $R_{\mathcal{E}}(x, y)<+\infty$. Then $Y$ is decomposed into a finite number of the equivalence classes $Y_{\alpha}(\alpha=1, \ldots, p)$; each class $Y_{\alpha}$ is open and closed in $Y$. Moreover for each $\alpha$ and large $n$, the inverse image of $Y_{\alpha}$ by $f_{n}, X_{n ; \alpha}=f_{n}^{-1}\left(Y_{\alpha}\right)$, is open and closed in $X_{n}$ and one has

$$
\begin{gathered}
\lim _{n \rightarrow \infty} \sup \left\{\left|R_{n}(x, y)-R_{\mathcal{E}}\left(f_{n}(x), f_{n}(y)\right)\right| \mid x, y \in X_{n ; \alpha}\right\}=0 ; \\
\lim _{n \rightarrow \infty} \sup _{y \in Y_{\alpha}} R_{\mathcal{E}}\left(f_{n}\left(X_{n ; \alpha}\right), y\right)=0 .
\end{gathered}
$$


(vi) Let $\chi_{\alpha}$ be the characteristic function of the subspace $Y_{\alpha}(1 \leq \alpha \leq p)$. Then $\chi_{\alpha} \in D[\mathcal{E}]$ and $\mathcal{E}\left(\chi_{\alpha}, u\right)=0$ for all $u \in D[\mathcal{E}]$, and if $\mathcal{E}(u, u)=0$, then $u$ is a linear combination of the characteristic functions $\chi_{\alpha}(1 \leq \alpha \leq p)$. Moreover let $D\left[\mathcal{E}_{\alpha}\right]=\left\{u \in D[\mathcal{E}] \mid \operatorname{supp} u \subset Y_{\alpha}\right\}$ and $\mathcal{E}_{\alpha}(u, v)=\mathcal{E}(u, v)\left(u, v \in D\left[\mathcal{E}_{\alpha}\right]\right)$. Then one has $D[\mathcal{E}]=\sum_{\alpha=1}^{p} D\left[\mathcal{E}_{\alpha}\right]$ and $\varepsilon(u, v)=\sum_{\alpha=1}^{p} \varepsilon_{\alpha}\left(\chi_{\alpha} u, \chi_{\alpha} v\right)$

(vii) Another equivalence relation $\sim_{0}$ is defined on $Y$ by $x \sim_{0} y$ if and only if $R_{\varepsilon}(x, y)=0$. Let $Y^{*}=Y / \sim_{0}$ and $Y_{\alpha}^{*}=Y_{\alpha} / \sim_{0}(\alpha=1, \ldots, p)$ be respectively the quotient spaces of $Y$ and $Y_{\alpha}$. Then for each $\alpha, R_{\mathcal{E}}$ provides $Y_{\alpha}^{*}$ a distance $R_{\alpha}$ which induces the same topology as the original one, and $D\left[\mathcal{E}_{\alpha}\right]$ is included in the pull-back of $C\left(Y_{\alpha}^{*}\right)$ by the canonical projection $\rho_{\alpha}$ of $Y_{\alpha}$ onto $Y_{\alpha}^{*}$. Thus the form $\varepsilon_{\alpha}$ can be assumed to be defined on $C\left(Y_{\alpha}^{*}\right) ;\left(\varepsilon_{\alpha}, D\left[\mathcal{E}_{\alpha}\right]\right)$ becomes a resistance form on $Y_{\alpha}^{*}$ and $R_{\alpha}$ is the associated resistance metric, that is,

$$
R_{\alpha}\left(x^{*}, y^{*}\right)=\sup \left\{\frac{\left|u\left(x^{*}\right)-u\left(y^{*}\right)\right|^{2}}{\mathcal{E}_{\alpha}(u, u)} \mid u \in D\left[\mathcal{E}_{\alpha}\right], \mathcal{E}_{\alpha}(u, u) \neq 0\right\}, \quad x^{*}, y^{*} \in Y_{\alpha}^{*} .
$$

Moreover a sequence of the compact metric spaces $\left(X_{n ; \alpha}, R_{n}\right)$ converges to $\left(Y_{\alpha}^{*}, R_{\alpha}\right)$ as $n \rightarrow \infty$ in the GromovHausdorff sense via the approximating maps $\rho_{\alpha} \circ f_{n}: X_{n ; \alpha} \rightarrow Y_{\alpha}^{*}$, and the form $\mathcal{E}_{X_{n ; \alpha}^{*}}^{*}$ on $C\left(X_{n ; \alpha}\right) \Gamma$-converges to $\mathcal{E}_{\alpha}$ as $n \rightarrow \infty$.

We remark that the compactness condition (iii) as above is indispensable in Theorem 3.4, and some results follows from the theorem.

Corollary 3.5. Let $\left\{K_{n}\right\}$ be a sequence of closed subspaces of compact metric graphs $G_{n}$ and suppose that the metric space $K_{n}$ with the induced Riemannian distance $d_{G_{n}}^{R}$ converges to a compact metric space $\left(X, d_{X}\right)$ in the GromovHausdorff sense via approximating maps $f_{n}: K_{n} \rightarrow X$. Then the resistance metric $R_{G_{n}}$ on $K_{n}$ converges to a continuous pseodo-distance $R$ on $X$ with respect to the Gromov-Hausdorff distance via the same approximating maps if and only if the resistance form $\mathcal{E}_{K_{n}}^{*} \Gamma$-converges to a lower semi-continuous functional $\&$ on $C(X)$ as $n \rightarrow \infty$. In these cases, one has

$$
0 \leq R(x, y) \leq d_{X}(x, y), \quad x, y \in X
$$

and $R$ is given by

$$
R(x, y)=\sup \left\{\frac{|u(x)-u(y)|^{2}}{\mathcal{E}(u, u)} \mid u \in D[\mathcal{E}], \mathcal{E}(u, u) \neq 0\right\}, \quad x, y \in X .
$$

Corollary 3.6. Let $\left\{\left(X_{n}, R_{n}\right)\right\}$ be a sequence of compact metric spaces of resistance forms $\mathcal{E}_{n}$ which converges to a compact metric space $\left(Y, R_{Y}\right)$ in the Gromov-Hausdorff sense via approximating maps $f_{n}: X_{n} \rightarrow Y$. Then the resistance form $\mathcal{E}_{K_{n}}^{*} \Gamma$-converges to a resistance form $\mathcal{E}$ on $C(X)$ which is associated with the limit distance $R_{Y}$.

In these corollaries, the definition of a resistance metric allows us to apply Ascoli-Arzelà's theorem to the sequences and see that the compactness condition (iii) holds true.

In the case where a sequence of networks $\left(V, E_{n}, r_{n}\right)$ with the same set of verticies $V$ is considered, letting $f_{n}$ as in Theorem 3.4 be the identity map of $V$, we see tha the compactness condition (iii) is always satisfied. Therefore we can apply our theorem to this case (cf. [4]).

\section{Spectral Embeddings and Spectral Convergence}

4.1 We consider a nonnegative quadratic form $(\mathcal{E}, D[\mathcal{E}])$ on a set $X$ satisfying the properties (RF-i), (RF-ii), (RF-iv) and that $R_{\mathcal{E}}(x, y)>0$ for all $x, y \in X$ with $x \neq y$. We suppose that the metric space $\left(X, \sqrt{R_{\mathcal{E}}}\right)$ is separable. Let $\mu$ be a Borel measure on $X=\left(X, \sqrt{R_{\mathcal{E}}}\right)$ with support $K$ such that

$$
\mu(X)(=\mu(K))<+\infty ; \int_{X} R_{\mathcal{E}}\left(x_{0}, x\right) d \mu(x)<+\infty
$$

for some $x_{0} \in X$ (and hence for any $x_{0}$ ). Denote the cardinality of the set $K$ by $N=\# K(\leq+\infty)$. Let $\left\{\lambda_{i} \mid 0=\lambda_{0}<\right.$ $\left.\lambda_{1} \leq \lambda_{2} \leq \cdots, 0 \leq i \leq N-1\right\}$ the set of eigenvalues of the self-adjoint operator $\mathcal{L}_{\mu}$ associated with the induced form $\mathcal{E}_{K}^{*}$ on $L^{2}(K, \mu)$ and $\Phi=\left\{\phi_{i} \mid 0 \leq i \leq N-1\right\}$ an orthonormal complete system of eigenfunctions. Recall that the effective resistance $R_{\mathcal{E}}$ is expressed on $K$ as follows:

$$
R_{\mathcal{E}}(x, y)=\sum_{i=1}^{N-1}\left(\frac{\phi_{i}(x)}{\sqrt{\lambda_{i}}}-\frac{\phi_{i}(y)}{\sqrt{\lambda_{i}}}\right)^{2}, \quad x, y \in K .
$$

In what follows, we understand $\phi_{i} / \sqrt{\lambda_{i}}=0$ for $i \geq N$ if $N$ is finite.

Let $\ell^{2}=\left\{\left(a_{i}\right) \mid \sum_{i=1}^{\infty} a_{i}^{2}<+\infty\right\}$, and define a map $I_{\Phi}$ of $K$ into $\ell^{2}$ by 


$$
I_{\Phi}(x)=\left(\frac{\phi_{i}(x)}{\sqrt{\lambda_{i}}}\right), \quad x \in K .
$$

Then $I_{\Phi}$ gives rise to an isometric embedding of $\left(K, \sqrt{R_{\mathcal{E}}}\right)$ into $\ell^{2}$. In fact, it satisfies the following properties:

$$
\begin{aligned}
& R_{G}(x, y)=\left\|I_{\Phi}(x)-I_{\Phi}(y)\right\|_{\ell^{2}}^{2}, \\
& g^{\mu}(x, y)=\left(I_{\Phi}(x), I_{\Phi}(y)\right)_{\ell^{2}}, \quad x, y \in K .
\end{aligned}
$$

Now we introduce another embedding of $K$ into $\ell^{2}$ as follows:

$$
J_{\Phi}(x)=\left(e^{-\lambda_{i} / 2} \phi_{i}(x)\right), \quad x \in K .
$$

This embedding has the following properties:

$$
\begin{aligned}
\left\|J_{\Phi}(x)-J_{\Phi}(y)\right\|_{\ell^{2}}^{2} & =\sum_{i=1}^{N-1} e^{-\lambda_{i}}\left(\phi_{i}(x)-\phi_{i}(y)\right)^{2} \\
& =p_{\mu}(1, x, x)-2 p_{\mu}(1, x, y)+p_{\mu}(1, y, y), \quad x, y \in K,
\end{aligned}
$$

where $p_{\mu}(t, x, y)$ denotes the kernel function of the semigroup $\exp \left(-t \mathcal{L}_{\mu}\right)$ generated by $\mathcal{L}_{\mu}$. Let

$$
S_{\mu}(x, y)=\sqrt{p_{\mu}(1, x, x)-2 p_{\mu}(1, x, y)+p_{\mu}(1, y, y)}, \quad x, y \in K .
$$

Then $S_{\mu}: K \times K \rightarrow[0,+\infty)$ is a distance on $K$ and $J_{\Phi}$ is an isometric embedding of the metric space $\left(K, S_{\mu}\right)$ into $\ell^{2}$. It is easy to see that

$$
0 \leq S_{\mu}(x, y) \leq R_{\mathcal{E}}(x, y)^{1 / 2}, \quad x, y \in K
$$

Given a function $u \in D\left[\mathcal{E}_{K}^{*}\right]$, we simply denote by $\tilde{u}$ the minimizer $H_{K ; u}$ in the set $\left\{v \in D\left[\mathcal{E}_{X}\right] \mid v_{\mid K}=u\right\}$ (see Section 1). Correspondingly we denote by $\widetilde{I}_{\Phi}, \widetilde{J}_{\Phi}, \widetilde{p}_{\mu}$ and $\widetilde{S}_{\mu}$ respectively the continuous extentions of the embeddings $I_{\Phi}, J_{\Phi}$, the kernel function $p_{\mu}$ and the distance $S_{\mu}$. We note that the extended maps $\widetilde{I}_{\Phi}$ and $\widetilde{J}_{\Phi}$ may not be injective on $X$ and hence $\widetilde{S}_{\mu}$ may be degenerate somewhere on $X$.

4.2 Now consider the same situation as in Theorem 3.4 and let $\left\{\left(X_{n}, R_{n}\right)\right\},\left(Y, R_{Y}, \mathcal{E}\right), f_{n}: X_{n} \rightarrow Y$ and $h_{n}: Y \rightarrow X_{n}$ be as before. Let us take a sequence of Radon measures $\mu_{n}$ on $X_{n}$ with supp $\mu_{n}=X_{n}$ such that the push-forward measure $f_{n *} \mu_{n}$ weakly converges to a Radon measure $\mu$ on $Y$ as $n \rightarrow \infty$. Let $\mathcal{L}_{\mu}$ be the Laplacian in $L^{2}(K, \mu)$, where $K=\operatorname{supp} \mu$, associated with the induced form $\mathcal{E}_{K}^{*}$ on $C(K)$ and $\widetilde{p}_{\mu}$ the continuous extension of the kernel function $p_{\mu}$ of the semigroup generated by $\mathcal{L}_{\mu}$.

To state a result on spectral convergence, we need some definitions. We define a linear map $T_{n}: D\left[\mathcal{E}_{K}^{*}\right] \rightarrow L^{2}\left(X_{n}, \mu_{n}\right)$ by $T_{n}(u)=f_{n}^{*} \tilde{u}\left(=f_{n}^{*} H_{K ; u}\right), \quad u \in D\left[\mathcal{E}_{K}^{*}\right]$. A sequence of functions $u_{n} \in L^{2}\left(X_{n}, \mu_{n}\right)$ is said to $L^{2}$ strongly (resp. $L^{2}$ weakly) converge to a function $u \in L^{2}(K, \mu)$ if there exists a sequence of functions $v_{i}$ in $D\left[\mathcal{E}_{K}^{*}\right]$ such that $\lim _{i \rightarrow \infty} \limsup _{n \rightarrow \infty}\left\|T_{n}\left(v_{i}\right)-u_{n}\right\|_{L^{2}\left(X_{n}, \mu_{n}\right)}=0$ (resp. if, for every $v \in L^{2}(K, \mu)$ and any sequence of $v_{n} \in L^{2}\left(X_{n}, \mu_{n}\right)$ which $L^{2}$-strongly converges to $\left.v, \lim _{n \rightarrow \infty}\left(u_{n}, v_{n}\right)_{L^{2}\left(X_{n}, \mu_{n}\right)}=(u, v)_{L^{2}(K, \mu)}\right)(c f$. [21]).

Theorem 4.1. Under the conditions above, the following assertions hold:

(i) Let $\left\{u_{n}\right\}$ be a sequence of functions $u_{n} \in L^{2}\left(X_{n}, \mu_{n}\right)$ with $\sup _{n}\left\|u_{n}\right\|_{L^{2}\left(X_{n}, \mu_{n}\right)}<+\infty$ which $L^{2}$-weakly converges to a function $u \in L^{2}(K, \mu)$. Then for any $\alpha>0, w_{n}=\mathcal{R}_{\mu_{n} ; \alpha} u_{n}$ uniformly converges to $w=\left(\mathcal{R}_{\mu ; \alpha} u\right)^{\sim}$ and $\mathcal{E}_{n}\left(w_{n}, w_{n}\right)$ tends to $\mathcal{E}_{K}^{*}(w, w)$ as $n \rightarrow \infty$, where $\mathcal{R}_{\mu_{n} ; \alpha}$ and $\mathcal{R}_{\mu ; \alpha}$ are respectively the resolvents of the Laplacians $\mathcal{L}_{\mu_{n}}$ and $\mathcal{L}_{\mu}$.

(ii) The maps $f_{n}$ and $h_{n}$ approximate the kernel functions $p_{\mu_{n}}$ and $p_{\mu}$ in such a way that

$$
\begin{gathered}
\lim _{n \rightarrow \infty} \sup _{t>0, x, y \in X_{n}} e^{-(t+1 / t)}\left|p_{\mu_{n}}(t, x, y)-\widetilde{p}_{\mu}\left(t, f_{n}(x), f_{n}(y)\right)\right|=0 \\
\lim _{n \rightarrow \infty} \sup _{t>0, a, b \in Y} e^{-(t+1 / t)}\left|p_{\mu_{n}}\left(t, h_{n}(a), h_{n}(b)\right)-\widetilde{p}_{\mu}(t, a, b)\right|=0 \\
\lim _{n \rightarrow \infty} \sup _{a \in Y} \widetilde{S}_{\mu}\left(a, f_{n} \circ h_{n}(a)\right)=0 .
\end{gathered}
$$

(iii) For each $i$, the $i$-th eigenvalue of $\mathcal{L}_{\mu_{n}}$ converges to that of $\mathcal{L}_{\mu}$ in the sense that if $N=\# K<+\infty$ and $i \geq N$, then the $i$-th eigenvalue diverges to infinity as $n \rightarrow \infty$.

Example Let $(V, E, r)$ be a finite network and $\mathcal{E}$ the associated form on $\ell(V)$. Given a sequence of positive functions $\pi_{n}$ on $V$, we have a sequence of Morkovian forms $\mathcal{E}_{\pi_{n}}$ on $\ell(V)$ defined by

$$
\mathcal{E}_{\pi_{n}}(u, v)=\mathcal{E}\left(u / \pi_{n}, v / \pi_{n}\right), \quad u, v \in \ell(V) .
$$

Suppose that $\pi_{n}$ converges to a nonnegative function $\pi_{\infty}$ on $V$. Let $\mu_{n}$ (resp. $\mu_{\infty}$ ) be measures on $V$ given by $\mu_{n}(u)=\sum_{x \in V} u(x) \pi_{n}(x)^{2}$ (resp. $\left.\mu_{\infty}(u)=\sum_{x \in K} u(x) \pi_{\infty}(x)^{2}\right)$, where $K$ stands for the support of $\pi_{\infty}$. Then as $n \rightarrow \infty$, the Laplacian $\mathcal{L}_{\mu_{n}}$ of $\mathscr{E}$ in $L^{2}\left(V, \mu_{n}\right)$ converges to the Laplacian $\mathcal{L}_{\mu_{\infty}}$ of the induced form $\mathcal{E}_{K}^{*}$ in $L^{2}\left(K, \mu_{\infty}\right)$ in the sense 
of Theorem 4.1. Moreover the form $\varepsilon_{\pi_{n}} \Gamma$-converges to a Markovian form $\left(\varepsilon_{\infty}, D\left[\varepsilon_{\infty}\right]\right)$ on $\ell(V)$ defined by $D\left[\varepsilon_{\infty}\right]=$ $\left\{H_{K ; u} \mid u \in \ell(K)\right\}$ and $\mathcal{E}_{\infty}\left(H_{K ; u}, H_{K ; u}\right)=\mathcal{E}\left(H_{K ; u}, H_{K ; u}\right)$.

4.3 Given two positive constants $D$ and $M$, let $B(D, M)=\left\{\left(a_{i}\right) \in \ell^{2} \mid \sum_{i=1}^{\infty}(1+i / D M) a_{i}^{2} \leq 4 D^{2} M\right\}$. Then it is a compact subspace of $\ell^{2}$.

Now we consider a nonnegative quadratic form $(\mathcal{E}, D[\mathcal{E}])$ on a set $X$ such that it satisfies the properties (RF-i), (RF-ii) and (RF-iv), $R_{\mathcal{E}}(x, y)>0$ for all $x, y \in X$ with $x \neq y$, and the metric space $\left(X, \sqrt{R_{\mathcal{E}}}\right)$ is compact. Let us denote by $\wp_{D, M}$ a set of such triplets $\left(X, \mathcal{E}, \sqrt{R_{\mathcal{E}}}\right)$ endowed with Radon measures $\mu$ such that

$$
\operatorname{diam}\left(X, \sqrt{R_{\mathcal{E}}}\right) \leq D<+\infty, \operatorname{supp} \mu=X, \mu(X) \leq M .
$$

Then in view of (3), we see that the image $J_{\Phi}(X)$ of the isometric embedding $J_{\Phi}$ described in the last section is included in the compact subspace $B(D, M)$ of $\ell^{2}$.

Recall here that the set of closed subspaces of a compact metric space is indeed compact with respect to the Hausdorff distance on the set (cf. e.g., [1]). It suggests us that the set $\S_{D, M}$ is precompact in a sense from a view-point of Laplacians ( $c f .[15,19])$. In fact, we have the following.

Theorem 4.2. Let $\left\{\left(X_{n}, \mathcal{E}_{n}, \mu_{n}\right)\right\}$ be a sequence of $\S_{D, M}$. Then there exist a subsequence $\left\{\left(X_{m}, \mathcal{E}_{m}, \mu_{m}\right)\right\}$, a compact metric space $(\hat{X}, \hat{S})$, a nonnegative quadratic form $(\hat{\mathcal{E}}, D[\hat{\mathcal{E}}])$ on $\hat{X}$ satisfying $(R F-i),(R F-i i)$ and $(R F-i v)$, and a Radon measure $\mu$ on $\hat{X}$ such that

(i) the sequence of compact metric spaces $\left(X_{m}, S_{\mu_{m}}\right)$ converges to $(\hat{X}, \hat{S})$ in the Gromov-Hausdorff sense via approximating maps $f_{m}: X_{m} \rightarrow \hat{X}$ and $h_{m}: \hat{X} \rightarrow X_{m}$;

(ii) the sequenec of the forms $\mathcal{E}_{m} \Gamma$-converges to $\hat{\mathcal{E}}$;

(iii) letting $\hat{R}(x, y)=\sup \left\{|u(x)-u(y)|^{2} / \hat{\mathcal{E}}(u, u) \mid u \in D[\hat{\mathcal{E}}], \hat{\mathcal{E}}(u, u) \neq 0\right\}$, one has

$$
\hat{S}(x, y) \leq \hat{R}(x, y)^{1 / 2} \leq \liminf _{m \rightarrow \infty} R_{m}\left(h_{m}(x), h_{m}(y)\right)^{1 / 2} \leq D, \quad x, y \in \hat{X} .
$$

(iv) the image measure $f_{m *} \mu_{m}$ weakly converges to $\mu$;

(v) by using a linear map $T_{m}: D\left[\mathcal{E}_{K}^{*}\right] \rightarrow L^{2}\left(X_{m}, \mu_{m}\right)$ defined as before, where $K=\operatorname{supp} \mu$, one has the same assertions as in Theorem 4.1.

Remarks (i) In view of the assertion (iii) above, the identity map of $\left(\hat{X}, \hat{R}^{1 / 2}\right)$ onto $(\hat{X}, \hat{S})$ is continuous, but not homeomorphic in general. In fact, $\left(\hat{X}, \hat{R}^{1 / 2}\right)$ may be noncompact. See Example below.

(ii) When each form $\mathcal{E}_{m}$ is Markovian, so is the limit form $\hat{\mathcal{E}}$, and hence in this case, $\hat{\mathcal{E}}$ is a resitance form on the set $\hat{X}$ and $D[\hat{\mathcal{E}}]$ is dense in $C(\hat{X}, \hat{S})$.

(iii) Given a closed subset $B$ of $(\hat{X}, \hat{S})$, we have the induced form $\left(\hat{\mathcal{E}}_{B}^{*}, D\left[\hat{\mathcal{E}}_{B}^{*}\right]\right)$ in $C(B)$; in particular, for any $w \in D\left[\hat{\mathcal{E}}_{B}^{*}\right]$, there exists a unique function $H_{B ; u} \in D[\hat{\mathcal{E}}]$ such that $\hat{\mathcal{E}}\left(H_{B ; u}, v\right)=0$ for all $v \in D[\hat{\mathcal{E}}]$ vanishing on $B$.

Example Let $\Gamma_{n}$ be a subgraph of $\mathbf{Z}^{d}$ generated by the set of vertices $V_{n}=\left\{\left(x_{1}, \ldots, x_{d}\right)|| x_{i} \mid \leq n, i=1, \ldots, d\right\}$. Then it is known that the effective resistance of $\Gamma_{n}$ satisfies

if $d=2$, and

$$
c_{2} \log n \leq \max \left\{R_{n}(x, y) \mid x, y \in V_{n}\right\} \leq C_{2} \log n
$$

$$
0<c_{d} \leq \min \left\{R_{n}(x, y) \mid x, y \in V_{n}, x \neq y\right\} \leq \max \left\{R_{n}(x, y) \mid x, y \in V_{n}\right\} \leq C_{d}
$$

if $d \geq 3$, where $c_{d}$ and $C_{d}$ are positive constants depending only on $d$ (cf. [2]).

Now we consider the case where $d \geq 3$ and a sequence of measures $\mu_{n}$ on $V_{n}$ defined by $\mu_{n}(u)=\sum_{x \in V_{n}} u(x) \pi(x)^{2}$ $\left(u \in \ell\left(V_{n}\right)\right)$, where $\pi$ is a positive function on $\mathbf{Z}^{d}$ such that $\sum_{x \in \mathbf{Z}^{d}} \pi(x)^{2}<+\infty$. Then we have a compact metric space $\left(\mathbf{Z}^{d} \cup\{\infty\}, \hat{S}, \mu\right)$ to which the sequence of $G_{n}$ with the measure $\mu_{n}$ convegres in the sense of Theorem 4.1. However the effective resistance of $\mathbf{Z}^{d} \cup\{\infty\}$ provides it the discrete topology.

\section{Royden's Compactification of an Infinite Network}

In the last example, the one-point compactification of the lattice $\mathbf{Z}^{d}$ turns out to be the Royden compactification of $\mathbf{Z}^{d}$ if $d \geq 3$. Relevant to this example, we consider Royden's compactification of a locally finite, connected and infinite network. We refer the reader to e.g., Soardi [26] and the references therein for some basic properties and results on the compactification of networks, and Sario-Nakai [25] for the case of a Riemannian manifold. See also Saloff-Coste [23] and the references therein for some related topics and open questions.

5.1 Let $\Gamma=(V, E, r)$ be a locally finite, connected and infinite networks and $\left(\varepsilon_{V}^{*}, D\left[\varepsilon_{V}^{*}\right]\right)$ its resistance form. In what follows, we write $\left(\varepsilon_{\Gamma}, D[V]\right)$ instead of $\left(\mathcal{E}_{V}^{*}, D\left[\mathcal{E}_{V}^{*}\right]\right)$ and denote by $B D[V]$ the space of bounded functions in $D[V]$ which is endowed with the norm: $\|u\|_{B D}=\sqrt{\mathcal{E}_{\Gamma}(u, u)}+\sup _{x \in V}|u(x)|$. Associated to the Banach algebra $B D[V]$ with unit element 1 , we have a compactification of $V$, called the Royden compactification of the network, that is described as follows: there exists a unique (up to homeomorphisms) compact Hausdorff space $\mathfrak{R}_{\Gamma}$ such that $\mathfrak{R}_{\Gamma}$ contains $V$ as an open dense subset and every function in $B D[V]$ can be continuously extended to $\Re_{\Gamma}$ and $B D[V]$ separates points in $\Re_{\Gamma}$. 
The compact set $\partial \Re_{\Gamma}=\Re_{\Gamma} \backslash V$ is called the Royden boundary of the network and a distinguished part of the Royden boundary, called the harmonic boundary of the network, is defined by

$$
\Delta_{V}=\left\{x \in \partial \Re_{\Gamma} \mid f(x)=0 \text { for all } f \in B D_{0}[V]\right\},
$$

where $D_{0}[V]$ is the closure of the space of finitely supported functions in $\left(D[V], \sqrt{\mathcal{E}_{\Gamma}+\delta_{o}^{2}}\right)$. Here we recall the fact that for any $u \in C\left(\Delta_{\Gamma}\right)$, there is a unique harmonic function $h$ on $V$ such that for all $p \in \Delta_{\Gamma}, \lim _{x \in V \rightarrow p} h(x)=u(p)$, and if $u \in D[V]$, then $h$ is a unique energy minimizer among functions in $D[V]$ with the same boundary value as $u$ (cf. [26], Chap. VI).

Now as in Section 4, let us consider a measure $\mu=\sum_{x \in V} \pi(x)^{2} \delta_{x}$ on $V$ such that $\pi(x)>0$ for all $x \in V, \mu(V)=$ $\sum_{x \in V} \pi(x)^{2}<+\infty$, and $\int_{V} R_{\Gamma}(o, y) d \mu(y)<+\infty$ for some $o \in V$, where $R_{\Gamma}$ denotes the effective resistance of the network. Let $\Phi=\left\{\phi_{i} \mid i=0,1,2, \ldots\right\}$ be an orthonormal complete system of eigenfunctions of the Laplace operator $\mathcal{L}_{\mu}$ acting on $L^{2}(V, \mu)$ and $J_{\Phi}: V \rightarrow \ell^{2}$ the isometric embedding of the metric space $\left(V, S_{\mu}\right)$ into $\ell^{2}$ as in Section 4 . We denote by $\bar{V}_{\mu}$ the completion of $\left(V, S_{\mu}\right)$. Then $\bar{V}_{\mu}$ is compact if the effective resistance $R_{\Gamma}$ is bounded in $V$, as seen in Section 4. Moreover in this case, setting

$$
D\left[\bar{V}_{\mu}\right]=\left\{u \in C\left(\bar{V}_{\mu}\right) \mid u_{\mid V} \in D[V]\right\}, \quad \bar{\varepsilon}_{V}(u, u)=\mathcal{E}_{V}\left(u_{\mid V}, u_{\mid V}\right), \quad u \in D\left[\bar{V}_{\mu}\right],
$$

we have a resistance form $\left(\bar{\varepsilon}_{\Gamma}, D\left[\bar{V}_{\mu}\right]\right)$ on $\bar{V}_{\mu}$. The topology induced from the resistance metric may be strictly finer than that of the distance $S_{\mu}$, as is noted in the last example.

Now we state the following

Theorem 5.1. Let $\Gamma=(V, E, r)$ be a locally finite, connected and infinite network. Suppose that the effective resistance of the network is uniformly bounded in $V$. Then the following assertions hold:

(i) The spaces $B D[V]$ and $D[V]$ coincide and further the norms are equivalent.

(ii) Given a measure $\mu$ on $V$ as above, the metric space $\left(\bar{V}_{\mu}, S_{\mu}\right)$ is homeomorphic to the Royden compactification of the network.

(iii) $\Delta_{\Gamma}=\partial \Re_{\Gamma}$.

As a result of the last assertion (iii), we see that if in addition any harmonic function of bounded energy on $V$ must be constant, then the Royden boundary of $V$ consists of a single point.

It would be interesting to give sufficient conditions on a network under which the resistance metric has bounded diameter ( $c f$. Corollary 2.5).

Let $\Gamma=(V, E)$ be a locally finite, connected graph with all the resistances equal to 1 . Assume that $\sup _{x \in V} \operatorname{deg}(x)<$ $+\infty$ and there exists a positive constant $\lambda$ such that

$$
\lambda \sum_{x \in V} u(x)^{2} \leq \mathcal{E}_{V}(u, u), \quad u \in D_{0}[V]
$$

Then we have $\Delta_{V}=\partial \Re_{\Gamma}$, since $\Gamma$ is transient and $B D_{0}[V]=\left\{f \in B D[V] \mid f(x)=0\right.$ for all $\left.x \in \Delta_{V}\right\}$ (cf. [26], p. 144). It would be asked if such a graph of bounded degree and a positive spectral gap is of bounded diameter with respect to the resistance metric when the graph has only one end, that is, it is connected at infinity.

5.2 Let $(X, d),\left(X^{\prime}, d^{\prime}\right)$ be two metric spaces. A map $\psi: X \rightarrow X^{\prime}$ is a quasi-isometry from $X$ to $X^{\prime}$ if there are constants $C_{1}, \ldots, C_{5}$ such that:

(i) For all $x^{\prime} \in X^{\prime}$, there exists $x \in X$ such that $d^{\prime}\left(x^{\prime}, \psi(x)\right) \leq C_{1}$;

(ii) For all $x, y \in X, C_{2} d(x, y)-C_{3} \leq d^{\prime}(\psi(x), \psi(y)) \leq C_{4} d(x, y)+C_{5}$.

A quasi-isometry is called in [13] and [14] a rough isometry.

In what follows, we consider locally finite, connected graphs $\Gamma=(V, E)$ of bounded degree with the geodesic distances $d_{\Gamma}^{R}$.

Theorem 5.2. Let $\Gamma=\left(V, E, d_{\Gamma}^{R}\right), \Gamma^{\prime}=\left(V^{\prime}, E^{\prime}, d_{\Gamma^{\prime}}^{R}\right)$ be two graphs of bounded degree, and suppose that there is a quasi-isometry $\psi: V \rightarrow V^{\prime}$. Then the following assertions hold:

(i) Relative to the resistance metrics of $\Gamma$ and $\Gamma^{\prime}$, the map $\psi$ induces a quasi-isometry (with different constants). In particular, the diameters on the resistance metrics are finite if so is either of them.

(ii) A quasi-isometry $\psi: V \rightarrow V^{\prime}$ is continuously extended to a map $\bar{\psi}$ from the Royden boundary of $\Gamma$, $\Re_{\Gamma}$, to that of $\Gamma^{\prime}, \Re_{\Gamma^{\prime}}$, in such a way that $\bar{\psi}$ sends homeomorphically the Royden boundary and the harmonic boundary of $\Gamma$ to those of $\Gamma^{\prime}$, respectively, that is, $\bar{\psi}\left(\partial \Re_{\Gamma}\right)=\partial \Re_{\Gamma^{\prime}}$ and $\bar{\psi}\left(\Delta_{\Gamma}\right)=\Delta_{\Gamma^{\prime}}$.

Let $\Gamma=(V, E, r)$ be a locally finite, connected and infinite network. Given a point $p$, we have a positive regular Borel measure $v_{p}$, called the harmonic measure on the Royden boundary supported in the harmonic boundary ( $c f$. [26], Chap. VI). In the same situation as in Theorem 5.3, it will be interesting to see how the image measure $\bar{\psi}_{*} v_{p}$ of the harmonic measure on $\Delta_{\Gamma}, v_{p}$, can be compared with that on $\Delta_{\Gamma^{\prime}}, v_{p^{\prime}}$, where $p \in V$ and $p^{\prime} \in V^{\prime}$. We would like also to know how we could characterize a map $\phi: V \rightarrow V^{\prime}$ which is continuously extended to a map $\bar{\phi}: \mathfrak{R}_{\Gamma} \rightarrow \mathfrak{R}_{\Gamma^{\prime}}$ in such a way that $\bar{\phi}$ induces a homeomorphism between the boundaries.

Now we say that a complete, connected Riemannian manifold $M$ is of bounded geometry if the Ricci curvature of $M$ 
is bounded below and the injectivity radius $\operatorname{inj}(M)$ of $M$ is bounded below by a positive constant. For such a Riemannian manifold $M$, we take a maximal set $W$ of $\varepsilon$-separated points, where $\varepsilon$ is a fixed positive number less than $\operatorname{inj}(M) / 2$. We say that there is an edge with endpoints $x, y \in W$ if $0<d_{M}^{R}(x, y) \leq 3 \varepsilon$, and the set of all such edges will be denoted by $F$. Then $N=(W, F)$ is a locally finite, connected graph of bounded degree and the inclusion $\iota$ of $W$ into $M$ induces a quasi-isometry between $\left(W, d_{N}^{R}\right)$ and $\left(M, d_{M}^{R}\right)$.

Let $\Gamma=(V, E)$ be a locally finite, connected graph of finite degree and assume that there exists a quasi-isometry $\psi$ from $V$ into $M$. Given a continuous function $f$ on $M$, let us define a function $f_{\psi}^{*}$ on $V$ by

$$
f_{\psi}^{*}(q)=\frac{1}{\operatorname{Vol}(B(\psi(q), 4 \varepsilon))} \int_{B(\psi(q), 4 \varepsilon)} f d v_{M}, \quad q \in V,
$$

and note that

$$
\mathcal{E}_{\Gamma}\left(f_{\psi}^{*}, f_{\psi}^{*}\right) \leq C \mathcal{E}_{M}(f, f)
$$

if $f$ has finite energy:

$$
\varepsilon_{M}(f, f)=\int_{M}|d f|^{2} d v_{M}<+\infty
$$

where $C$ is a positive constant independent of $f$ ( $c f$. [14]). Using this and a priori estimates on harmonic functions, we can prove the following

Theorem 5.3. Let $\Gamma=(V, E)$ and $M$ be respectively a locally finite, connected and infinite graph of bounded degree and a complete, connected Riemannian manifold of bounded geometry. Suppose that $\left(V, d_{\Gamma}^{R}\right)$ is quasi-isometric to $M=\left(M, d_{M}^{R}\right)$ and let $\psi: V \rightarrow M$ be a quasi-isometry. Then the following assertions hold:

(i) For any $u \in C\left(\Delta_{\Gamma}\right)$, there is a unique harmonic function $H$ on $M$ such that for all $p \in \Delta_{\Gamma}$, one has

$$
\lim _{x \in V \rightarrow p} H_{\psi}^{*}(x)=u(p)
$$

Moreover if u continuously extends to a function in $D[V]$, then the harmonic function $H$ has bounded energy and, letting $h$ be a unique harmonic function in $D[V]$ with the same boundary value $u$ as $H_{\psi}^{*}$, one has

$$
C^{-1} \mathcal{E}_{V}(h, h) \leq \mathcal{E}_{M}(H, H) \leq C \mathcal{E}_{V}(h, h),
$$

where $C$ is a positive constant independent of $u$.

(ii) For every harmonic function $H$ of finite energy on $M, H_{\psi}^{*}$ is extended to a continuous function on $\Delta_{\Gamma}$ with values in $\mathbf{R} \cup\{ \pm \infty\}$.

Theorem 5.3 implies that $\Gamma$ possesses no nonconstant harmonic functions of finite energy if and only if so does $M$. In fact, Holopainen and Soardi show in [12] that this holds true for $p$-harmonic finctions of finite $p$-Dirichlet energy, where $1<p<+\infty$. For a function $u$ on a locally finite, connected network $\Gamma=(V, E, r)$, we define a $p$-Dirichlet energy of $u$ by

$$
\mathcal{E}_{\Gamma}^{(p)}(u)=\frac{1}{2} \sum_{x \sim y} c(x, y)^{p-1}|u(x)-u(y)|^{p},
$$

where we set $c(x, y)=1 / r(x, y)$. Setting

$$
R_{\Gamma}^{(p)}(x, y)=\sup \left\{\frac{|u(x)-u(y)|^{p}}{\mathcal{E}_{\Gamma}^{(p)}(u)} \mid u \in \ell(V), \mathcal{E}_{\Gamma}^{(p)}(u)>0\right\}, x, y \in V,
$$

we might expect an analogue of Theorem 5.1 relative to $p$-Dirichlet energy.

Finally we consider a sequence of finite networks $\Gamma_{n}=\left(V_{n}, E_{n}, r_{n}\right)$ which satisfies the conditions in Theorem 3.4. Applying Theorem 3.3 to a sequence of the functionals $\mathcal{E}_{\Gamma_{n}}^{(p)}$, we have lower semi-continuous functionals $\mathcal{E}^{(p)}$ on the space of continuous functions of the limit space $Y$ in Theorem 3.4, which are $\Gamma$-limits of $\left\{\mathcal{E}_{\Gamma_{n}}^{(p)}\right\}$. We are interested in the limit functionals; it may occur that $\mathcal{E}^{(p)}$ is trivial.

\section{REFERENCES}

[1] Burago, D., Burago, Y., and Ivanov, S., A Course in Metric Geometry, GSM 33, Amer. Math. Soc., Providence, R.I., 2001.

[2] Chandra, A. K., Raghavan, P., Ruzzo, W. L., Smolensky, R., and Tiwari, P., "The electrical resistance of a graph captures its commute and cover times," Comput. Complex., 6: 312-340 (1996/1997).

[3] Cheeger, J., Degeneration of Einstein metrics and metrics with special holonomy, Surveys in Differential Geometry, VIII, 2973, International Press, 2003.

[4] Colin de Verdière, Y., Pan, Y., and Ycart, B., "Singular limits of Schrödinger operators and Markov processes," J. Operator Theory, 41: 151-173 (1999).

[5] Dal Maso, G., An Introduction to $\Gamma$-Convergence, Progress in Nonlinear Differential Equations and Their Applications 8, Birkäuser, Boston, 1993. 
[6] Eells, J., and Fuglede, B., Harmonic Maps between Riemannian Polyhedra, Cambridge University Press, Cambridge, 2001.

[7] Foster, R. M., The average impedance of an electrical network, Contributions to Applied Mechanics (Reissner Anniversary Volume), Edwords Bros., Ann Arbor, Mich. 333-340.

[8] Fukaya, K., Hausdorff convergence of Riemannian manifolds and its applications, in Recent Topics in Differential and Analytic Geometry, 143-238, Adv. Stud. in Pure Math. 18, North-Holland, Kinokuniya, Amsterdam, Tokyo, 1990.

[9] Fukushima, M., Oshima, Y., and Takeda, M., Dirichlet Forms and Symmetric Markov Processes, Walter de Gruyter, BerlinNew York, 1994.

[10] Gromov, M., Structures métriques pour les variétés riemanniennes, rédigé par J. LaFontaine et P. Pansu, Cedic FernandNathan, Paris, 1981.

[11] Gromov, M., Metric Structures for Riemannian and non-Riemannian spaces, (with appendices by M. Katz, P. Pansu, and S. Semmes, ed. by J. LaFontaine and P. Pansu, English translation by S. M. Bates), Progress in Mathematics 152, Birkhäuser, Boston, 1999.

[12] Holopainen, I., and Soardi, P. M., "p-harmonic functions on graphs and manifolds," Manuscripta Math., 94: 95-110 (1997).

[13] Kanai, M., "Rough isometries and the combinatorial arroximations of geometries of noncompact Riemannian manifolds," $J$. Math. Soc. Jpn., 37: 391-413 (1985).

[14] Kanai, M., "Rough isometries and the parabolicity of Riemannian manifolds," J. Math. Soc. Jpn., 38: $227-238$ (1986).

[15] Kasue, A., Convergence of Riemannian manifolds and Laplace operators, I, Ann. l'Institut Fourier, 52 (2002); — II, to appear in Potential Analysis.

[16] Kasue, A., "Convergence of metric measure spaces and energy forms," Amer. Math. Soc. Transl., 215: $79-96$ (2005).

[17] Kasue, A., "Convergence of metric graphs and energy forms," in preparation.

[18] Kasue, A., "Dirichlet finite harmonic functions and points at infinity of a network," in preparation.

[19] Kasue, A., and Kumura, H., "Spectral convergence of Riemannian manifolds," Tōhoku Math. J., 46: 147-179 (1994); — II, Tōhoku Math. J., 48: 71-120 (1996).

[20] Kigami, J., Analysis on Fractals, Cambridge University Press, Cambridge, 2001.

[21] Kuwae, K., and Shioya, T., "Convergence of spectral structures: a functional analytic theory and its appications to spectral geometry," Comm. Anal. Geom., 11: 599-673 (2003).

[22] Nakajima, H., "Convergence theorem of Einstein metrics and ALE spaces (Japanese)," Sūgaku, 44: 133-146 (1992).

[23] Saloff-Coste, L., Analysis on Riemannian co-compact covers, Surveys in Differential Geometry IX, 351-384, International Press, 2004.

[24] Sakai, T., Riemannian manifolds with bounded Ricci curvature and their limits (Japanese), "Riemmanian manifolds and their limits," Sūgaku Memoire, Math. Soc. of Japan, 2004.

[25] Sario, L., and M., Nakai, Classification theory of Riemann surfaces, Springer Verlag, Berlin-Hidelberg-New York, 1970.

[26] Soardi, P. M., Potential theory on infinite networks, Lec. Notes in Math. 1590, Springer, 1994.

[27] Tetali, P., "Random walks and effective resistance of networks," J. Theoret. Probability 4: 101-109 (1991).

[28] Yamaguchi, T., "The development of convergence theory for Riemannian manifolds (Japanese)," Sūgaku, 47: 46-61 (1995).

[29] Yamaguchi, T., “Collapsing Riemannian 4-manifolds (Japanese)," Sūgaku, 52: 172-186 (2000). 